\title{
Epidural-Related Fever and Maternal and Neonatal Morbidity: A Systematic Review and Meta-Analysis
}

\author{
Sophie Jansen ${ }^{a}$ Enrico Lopriore ${ }^{a} \quad$ Christiana Naaktgeboren $^{b}$ Marieke Sueters ${ }^{c}$

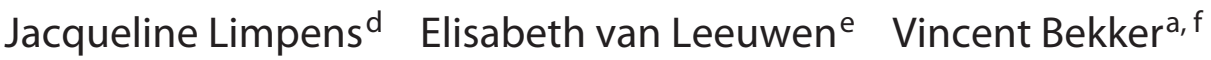 \\ ${ }^{a}$ Department of Neonatology, Leiden University Medical Center (LUMC), Leiden, The Netherlands; ${ }^{\text {b } U n i v e r s i t y ~}$ \\ Medical Center Utrecht (UMCU), Department of Data Management and Research Support, Julius Center for Health \\ Science and Primary Care, Utrecht, The Netherlands; ${ }^{C}$ Department of Obstetrics and Gynecology, Leiden University \\ Medical Center (LUMC), Leiden, The Netherlands; ${ }^{\mathrm{d}}$ Medical Library, Research Support, Amsterdam University

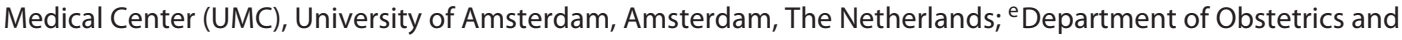

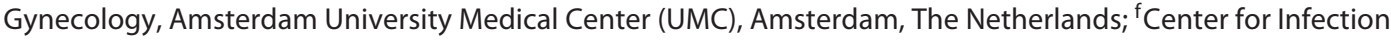 \\ and Immunity Amsterdam (CINIMA), Emma Children's Hospital, Amsterdam University Medical Center (UMC), \\ Amsterdam, The Netherlands
}

\section{Keywords}

Maternal fever · Epidural analgesia $\cdot$ Neonatal sepsis

\section{Abstract}

Background: While epidural analgesia (EA) is associated with maternal fever during labor, the impact on the risk for maternal and/or neonatal sepsis is unknown. Objectives: The aim of this systematic review was to investigate the effect of epidural-related intrapartum fever on maternal and neonatal outcomes. Methods: OVID MEDLINE, OVID Embase, the Cochrane Library, Cochrane Controlled Register of Trials, and clinical trial registries were searched for randomized controlled trials (RCT) and observational cohort studies from inception to November 2018. A total of 761 studies were identified with 100 eligible for full-text review. Only articles investigating the relationship between EA and maternal fever during labor were eligible for inclusion. Study quality was assessed using the Cochrane's Risk of Bias tool and National Institute of Health Quality Assessment Tool. Two meta-analyses - one each for the RCT and observational cohort groups - were performed using the random-effects model of Mantel-Haenszel to produce summary risk ratios (RR) with $95 \% \mathrm{Cl}$. Results: Twelve RCTs and 16 observational cohort studies involving 579,157 parturients were included. RRs for maternal fever for the RCT and cohort analyses were 3.54 (95\% Cl 2.61-4.81) and 5.60 (95\% Cl 4.50-6.97), respectively. Meta-analyses of RR for maternal infection in both groups were infeasible given few occurrences. Meta-analysis of data from observational studies showed an increased risk for maternal antibiotic treatment in the epidural group (RR 2.60; 95\% Cl 1.31-5.17). For both analyses, neonates born to women with an epidural were not evaluated more often for suspected sepsis. Neither analysis reported an increased rate of neonatal bacteremia or neonatal antibiotic treatment after EA, although data precluded conclusiveness. Conclusion: $\mathrm{EA}$ increases the risk of intrapartum fever and maternal antibiotic treatment. However, a definite conclusion on whether EA increases the risk for a proven maternal and/or neonatal bacteremia cannot be drawn due to the low quality of data. Further research on whether epidural-related intrapartum fever is of infectious origin or not is therefore needed.
(C) 2020 The Author(s)

Published by S. Karger AG, Basel karger@karger.com www.karger.com/neo

Karger $\stackrel{\text { ' }}{=}$

BOPEN ACCESS
(C) 2020 The Author(s)

Published by S. Karger AG, Basel

This article is licensed under the Creative Commons AttributionNonCommercial-NoDerivatives 4.0 International License (CC BYNC-ND) (http://www.karger.com/Services/OpenAccessLicense) Usage and distribution for commercial purposes as well as any distribution of modified material requires written permission.
Prof. Dr. Enrico Lopriore

Department of Neonatology

Leiden University Medical Center, PO Box 9600

NL-2300 RC Leiden (The Netherlands)

E-Mail e.lopriore@lumc.nl 


\section{Background}

Over the past 2 decades, epidural analgesia (EA) has evolved to become one of the most commonly used methods of pain relief during labor [1]. Despite its effectiveness as an analgesic, recent studies have suggested a strong causal relationship between EA and the development of intrapartum fever $\left(\geq 38^{\circ} \mathrm{C}\right)$ [2]. Because intrapartum fever is a possible indicator for neonatal morbidity and mortality, neonates of febrile parturients are often evaluated and treated for a presumed early-onset neonatal sepsis as a safeguard against a not otherwise specified intrapartum infection [3,4]. Unfortunately, the mechanisms by which intrapartum fever develops as a result of EA remain insufficiently understood [1]. Numerous studies demonstrate that maternal fever during labor is likely mediated by a noninfectious inflammatory component and that various circumstantial factors such as nulliparity, difficulty of labor, and prolonged ruptured membranes collectively contribute to its development [3]. The question as to whether epidural-related intrapartum fever is of infectious origin is therefore pertinent. We aim to evaluate the effect of EA on the rate of intrapartum fever, neonatal sepsis evaluations, maternal and/or neonatal bacteremia, and maternal and/or neonatal antibiotic treatment. Our interest mainly lies in examining whether maternal fever during labor increases the risk for neonatal and maternal sepsis as this will provide evidence about whether subsequent antibiotic treatment for a presumed perinatal infection is warranted. A systematic literature search with meta-analysis was performed to evaluate the available evidence on the effect of epidural-related intrapartum fever on adverse maternal and neonatal outcomes.

\section{Methods}

\section{Study Design}

This systematic review and meta-analysis is reported in accordance with the Preferred Reporting Items for Systematic Review and Meta-Analysis Protocols statement [5]. A protocol was registered in the PROSPERO international prospective register of systematic reviews (record number: CRD42017081244, available at www.crd.york.ac.uk.prospero).

\section{Sources and Study Selection}

The electronic databases OVID MEDLINE, OVID Embase, the Cochrane Library including the Cochrane Controlled Register of Trials, and the clinical trial registries ClinicalTrials.gov, trialregister.nl and clinicaltrialsregister.eu, were searched from inception to November 2018 using various controlled search terms (including MeSH-terms) and text words for labor EA with additional terms for antibiotics, fever, infection, or inflammation. Additionally, we searched for randomized controlled trials (RCT) comparing the effects of epidural versus intravenous/patient-controlled anesthesia to screen for data on fever and antibiotic use in full-text. No language or date restrictions were applied. Reference lists and cited publications from the selected articles were also cross-checked via Web of Science. All citations were imported into Endnote after which duplicate records were removed.

All RCTs and observational cohort studies investigating the relationship between EA and maternal fever during labor were eligible for inclusion. Case-control studies as well as systematic or literature reviews were excluded. After being initially selected based upon our primary outcome measure, studies reporting additional maternal and/or neonatal outcomes such as neonatal sepsis evaluations, maternal and/or neonatal bacteremia, and antibiotic treatment rates were also assessed. The primary outcome of interest is the development of maternal fever during labor after EA versus non-EA. Secondary outcomes include the incidence of neonatal sepsis work-ups, maternal and/or neonatal infection, and maternal and/or neonatal antibiotic treatment. All citations were independently screened at the title and abstract level and subsequently reviewed in full-text by 2 members of the review team (S.J. and V.B.).

\section{Data Extraction}

All data extraction was performed independently by 2 members of the review team (S.J. and V.B.) using the systematic review management tool Covidence (https://www.covidence.org). The following data were extracted from all selected articles: name of first author, journal and year of publication, geographical area, study design, sample sizes of each treatment arm, participant characteristics (parity, gestational age at delivery), and primary and secondary outcomes.

\section{Statistical Analysis}

Two meta-analyses, one for the RCT group and one for the observational cohort group, were performed and categorized by the type of outcome investigated. Data were meta-analyzed to calculate a summary risk ratio (RR), 95\% CI, and $p$ value. $p<0.05$ was regarded as significant. For each outcome, the individual effects and pooled mean effect size were summarized in a forest plot.

A random-effects model using Mantel-Haenszel's method for dichotomous data and DerSimonian and Laird's method for the $\mathrm{Tau}^{2}$ estimation was used. Heterogeneity of effect sizes was quantified using the $I^{2}$ statistic, representing the total variation across studies due to heterogeneity as opposed to random study sampling error. According to the Cochrane Handbook, no observed heterogeneity is denoted with a value of $0 \%$, while increasing heterogeneity is represented with larger values, where $<40 \%$ is low, $30-60 \%$ is moderate, $50-90 \%$ is substantial, and $75-100 \%$ is high [6]. A continuity correction of 0.5 was applied to all 4 cells of the contingency table for studies in which zero events were present in one or both treatment arms. Funnel plots were computed to assess the potential role of publication bias with standard error on the vertical axis and effect size on the horizontal axis. Studies in which publication bias is absent are expected to be distributed symmetrically around the pooled summary measure. In the presence of bias, smaller studies lacking "positive" results will be underrepresented, resulting in an asymmetrical distribution of the mean. Egger's regression test was performed to quantitatively assess the presence of funnel plot asymmetry. 
Fig. 1. Preferred Reporting Items for Systematic Review and Meta-Analysis Protocols flow chart of study selection procedure. RCT, randomized controlled trial.

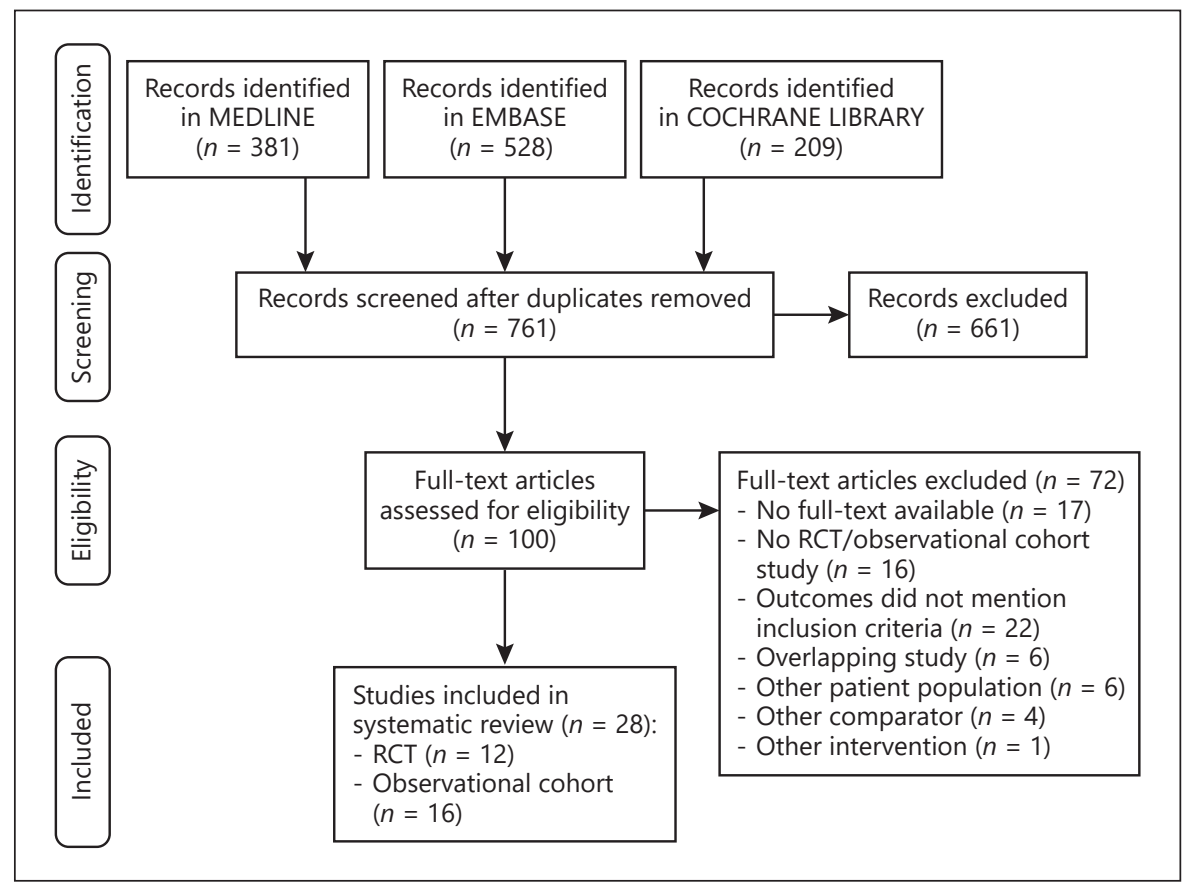

All statistical analyses were performed using the Meta and MetaFor packages of the research synthesis tool R (version 3.4.3, https://www.r-project.org).

\section{Risk of Bias and Quality of Evidence Assessment}

Two authors (S.J. and V.B.) independently assessed the methodological quality of each selected article using the Cochrane's risk-of-bias (RoB 2.0) tool [7] for RCTs and a modified version of the National Institute of Health Quality Assessment Tool for observational cohort studies [8]. The 5 criteria from the RoB tool included: bias arising from the randomization process, deviations from intended interventions, missing outcome data, inadequate measurement of the outcome, and selective reporting of the results. We slightly modified the National Institute of Health Quality Assessment Tool by broadening the number of criteria each study had to meet within the quality rating section to accommodate any potential concerns extending across multiple domains. Each RCT was rated as having either a "low," "high," or "unclear" RoB, while each cohort study was rated as having either a "good," "fair," "poor," or "very poor" overall quality. Any incongruities between the 2 reviewers was resolved by discussion and, where necessary, arbitration by a (fixed) third member of the review team. No study was excluded based on RoB.

The quality of evidence was independently assessed by 2 review authors (S.J. and V.B.) for each outcome within the RCT and observational cohort analyses using Cochrane's GRADE approach [9]. According to the GRADE methodology, the baseline quality rating for RCTs is "high" and for non-RCTs "low." A judgment was made based upon 5 quality assessment criteria, after which the ratings were either downgraded or upgraded $[9,10]$. In the presence of no serious concerns, the baseline quality was not downgraded. Evidence was downgraded 1 or 2 levels in the presence of a serious or very serious concern, respectively.

Epidural-Related Intrapartum Fever and Maternal and Neonatal Morbidity

\section{Results}

The study selection procedure is summarized in Figure 1. A total of 761 articles were identified by our search from inception to November 2018. After screening at the title and abstract level, 100 articles were deemed eligible for further review in full-text. Twenty-eight articles met the predetermined inclusion criteria. Main reasons for exclusion were ineligible study design, article not being available in full-text, and outcome measures outside our aim. Arbitration by the third member of the review team was not necessary for any of the included articles.

\section{Characteristics of Included Studies}

The main characteristics of each analyzed study are listed in Table 1. A total of 28 studies were included: 12 RCTs involving 5,137 parturients and 16 observational cohort studies involving 574,020 parturients. The majority of studies enrolled low-risk women with full-term pregnancies. Three studies [11-13] recruited low-risk women with preterm gestations, 1 study [14] recruited only women with an intermediate to high obstetric risk, and 1 study [15] consisted of parturients with pregnancyinduced hypertension. Ten studies enrolled only nulliparous women, while the remaining 18 enrolled women of mixed parity. All studies were written in the English language and over half were based in the United States of America (Table 1). 


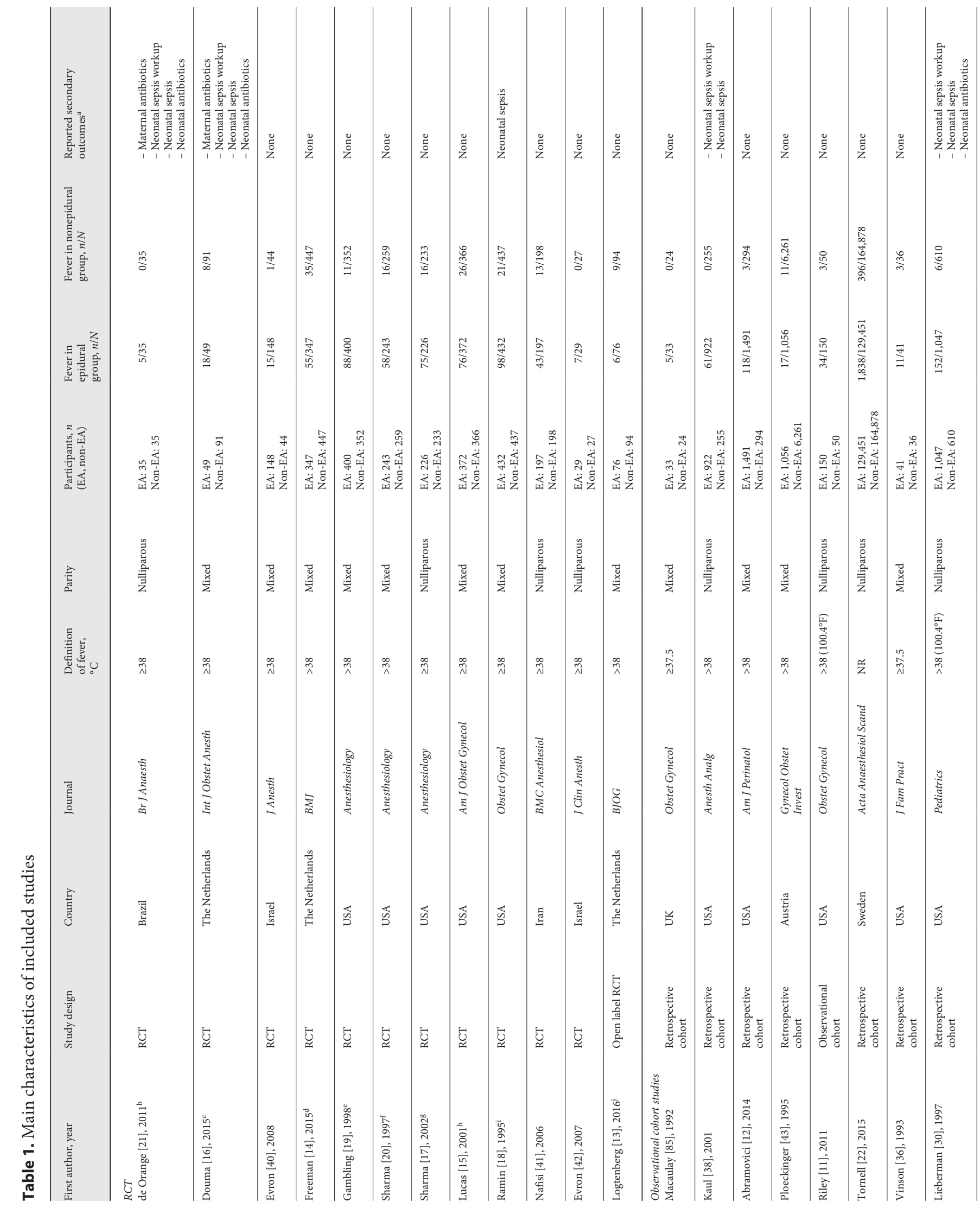



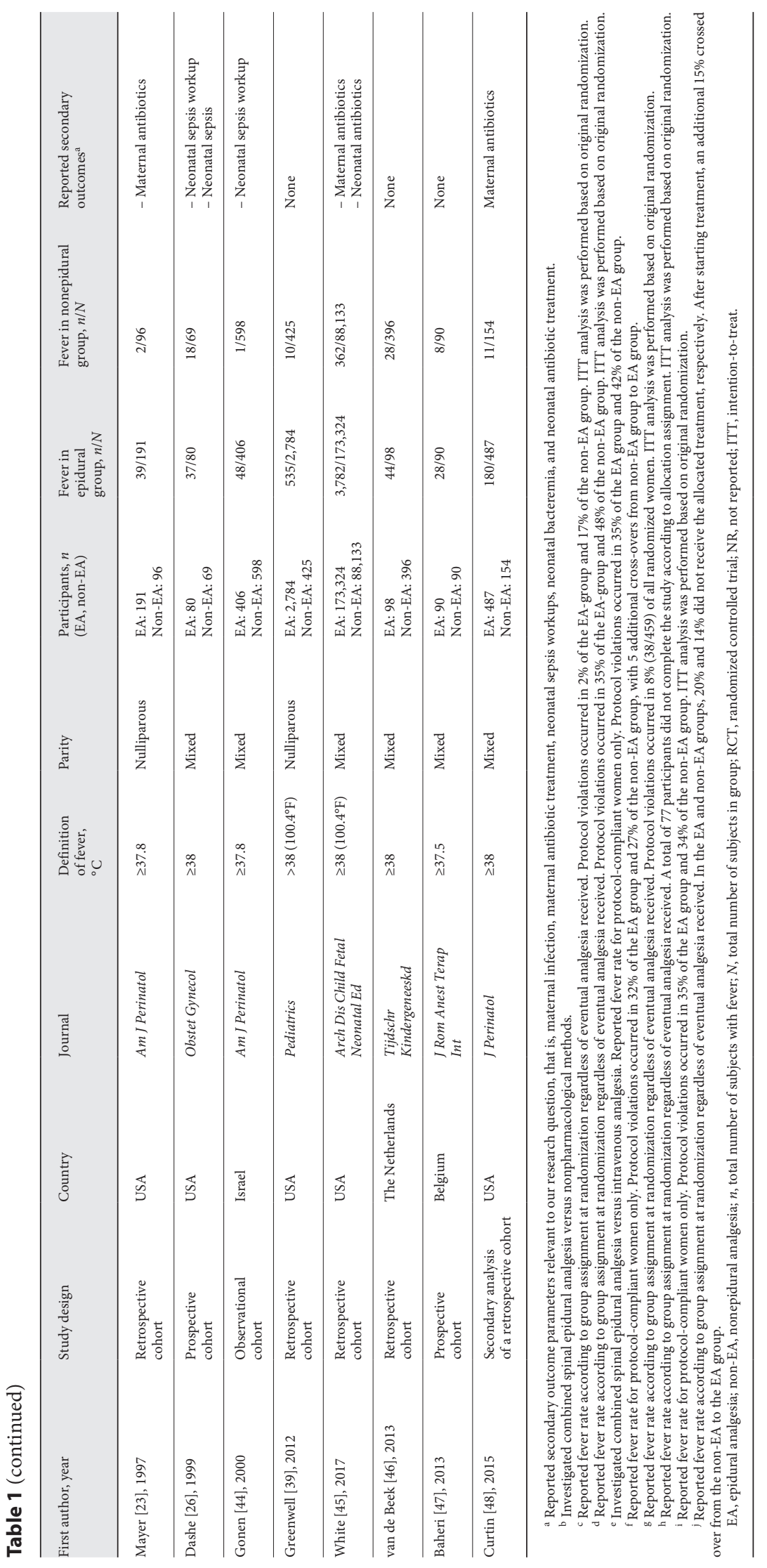


\section{Risk of Bias}

The RoB was considered "low" for 1 trial, with the remaining 11 trials annotated as "unclear". The 2 principle sources of bias were lack of blinding of participants and those delivering the intervention, as well as lack of adherence to the assigned intervention. Only 5 [14-18] of the 8 trials [13-20] in which a considerable amount of protocol deviations occurred implemented an appropriate corrective analysis (i.e., intention-to-treat analysis) to provide an unbiased estimate of the intervention effect. Quality assessment of the observational cohort studies revealed that 2 studies were of very poor quality, with 7 being of poor and another 7 of fair quality. Main sources of bias included lack of sample size justification, inadequate blinding of outcome assessors, and lack of information regarding dropout rate. Another important source of bias was the lack of adjustment for potential confounding factors (i.e., nulliparity, prolonged rupture of the membranes, and duration of labor), which may have led to a falsely positive association between EA, maternal fever, and maternal infection.

\section{Meta-Analysis of RCT}

Within the meta-analysis of the RCTs, women with EA were more likely to develop intrapartum fever compared with women without EA (RR 3.54; 95\% CI 2.61-4.81; $p<$ 0.0001; Fig. 2a). Maternal infection was studied in only 1 small RCT of 70 patients, in which no cases of confirmed maternal bacteremia were reported in both the epidural and the nonepidural groups [21]. Women with EA were over twice as likely to be treated with antibiotics postpartum (RR 2.09; 95\% CI 0.70-6.22; $p=0.18$; Fig. 2b), although this result is not statistically significant. The difference in diagnostic work-up of the neonate was not found to be different between the groups (RR 1.43; 95\% CI $0.43-4.78 ; p=0.56$; Fig. 2c). No association was found between EA and an increased risk of neonatal sepsis (RR 0.56 ; 95\% CI 0.08-3.91; $p=0.55$; Fig. 2 d) nor were infants born to women with EA more likely to be treated with antibiotics (RR 1.43; 95\% CI 0.43-4.78; $p=0.56$; Fig. 2e), although these conclusions are not entirely conclusive due to lack of precision of the estimates. Finally, the $I^{2}$ of $59 \%$ for the outcome maternal fever indicates a substantial amount of variation across studies.

\section{GRADE Assessment}

According to the GRADE assessment, the quality of evidence for the RCT analysis is very low for all secondary outcomes due to moderate risks of bias, general lack of precision of the estimate of effects, and likely presence of publication bias. The primary outcome has a similar very low quality of evidence, largely due to the high RoB and inconsistency of results as a result of inadequate blinding of treatment allocation and outcome accessors as well as substantial heterogeneity.

\section{Meta-Analysis of Observational Cohort Studies}

Within the cohort meta-analysis, the incidence of maternal fever during labor was again significantly higher in the epidural group compared to the nonepidural group (RR 5.60; 95\% CI 4.50-6.97; $p<0.0001$; Fig. 3a). None of the 3 studies $[11,22,23]$ that investigated maternal infection reported any proven sepsis cases via a positive blood culture. Women with EA were, however, more frequently treated with antibiotics (RR 2.60; 95\% CI 1.31-5.17; $p=0.0065$; Fig. $3 b)$. No difference in the risk of a diagnostic workup of the neonate was found between the groups (RR 2.64; 95\% CI 0.91-7.68; $p=0.07$; Fig. 3c), nor were neonates born to women with an epidural at a higher risk of being subjected to antibiotic treatment (RR 2.25; 95\% CI $0.73-6.69$; $p=0.15$; Fig. $3 \mathrm{~d}$ ). We also found no association between epidural use and the occurrence of neonatal bacteremia (RR 1.12; 95\% CI 0.47-2.69; $p=0.79$; Fig. 3e). In contrast to the RCT analysis, substantially larger degrees of heterogeneity were detected for the primary and most secondary outcomes, indicating substantial study variation due to potential confounding factors. Unfortunately, further sub-analyses regarding nulliparity and duration of ruptured membranes as an attempt to reduce the effect of confounding factors were infeasible due to lack of supporting data.

\section{GRADE Assessment}

According to the GRADE assessment, the quality of evidence for the observational cohort analysis is very low for 3 of the 4 secondary outcomes due to moderate risks of bias, substantial levels of heterogeneity, relatively weak precision of the estimate of effects, and possible presence of publication bias. Because of its narrower confidence interval, one secondary outcome (neonatal sepsis) was only downgraded 2 levels. Despite having a large effect size, the quality of evidence for the primary outcome can only be considered as very low due to the potential effects of confounding and substantial degree of heterogeneity.

\section{Publication Bias}

Funnel plots were created only for the primary outcome, but separately for the RCTs and observational cohort studies. Despite the apparent asymmetry in both funnel plots through visual inspection, formal testing us- 


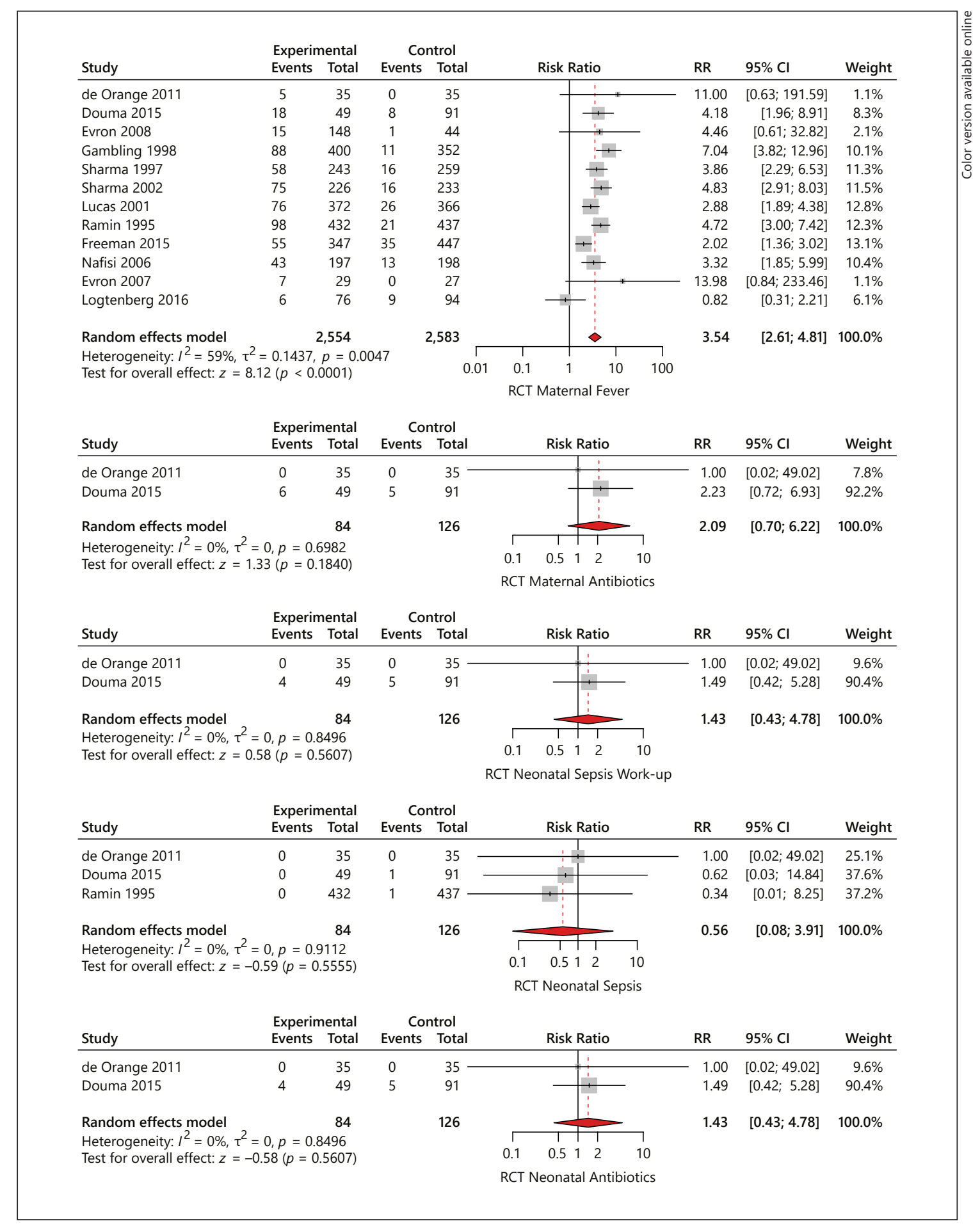

Fig. 2. Forest plots for RCT meta-analysis of primary and secondary outcomes epidural and non-epidural group. Size of gray boxes reflects precision or lack thereof of each individual study RR. Size of triangular random-effects summary measure reflects CI width. a RCT maternal fever. b RCT maternal antibiotics. c RCT neonatal sepsis work-up. d RCT neonatal sepsis. e RCT neonatal antibiotics. RR, risk ratio; RCT, randomized controlled trial.

Epidural-Related Intrapartum Fever and Maternal and Neonatal Morbidity
Neonatology 2020;117:259-270

DOI: $10.1159 / 000504805$ 


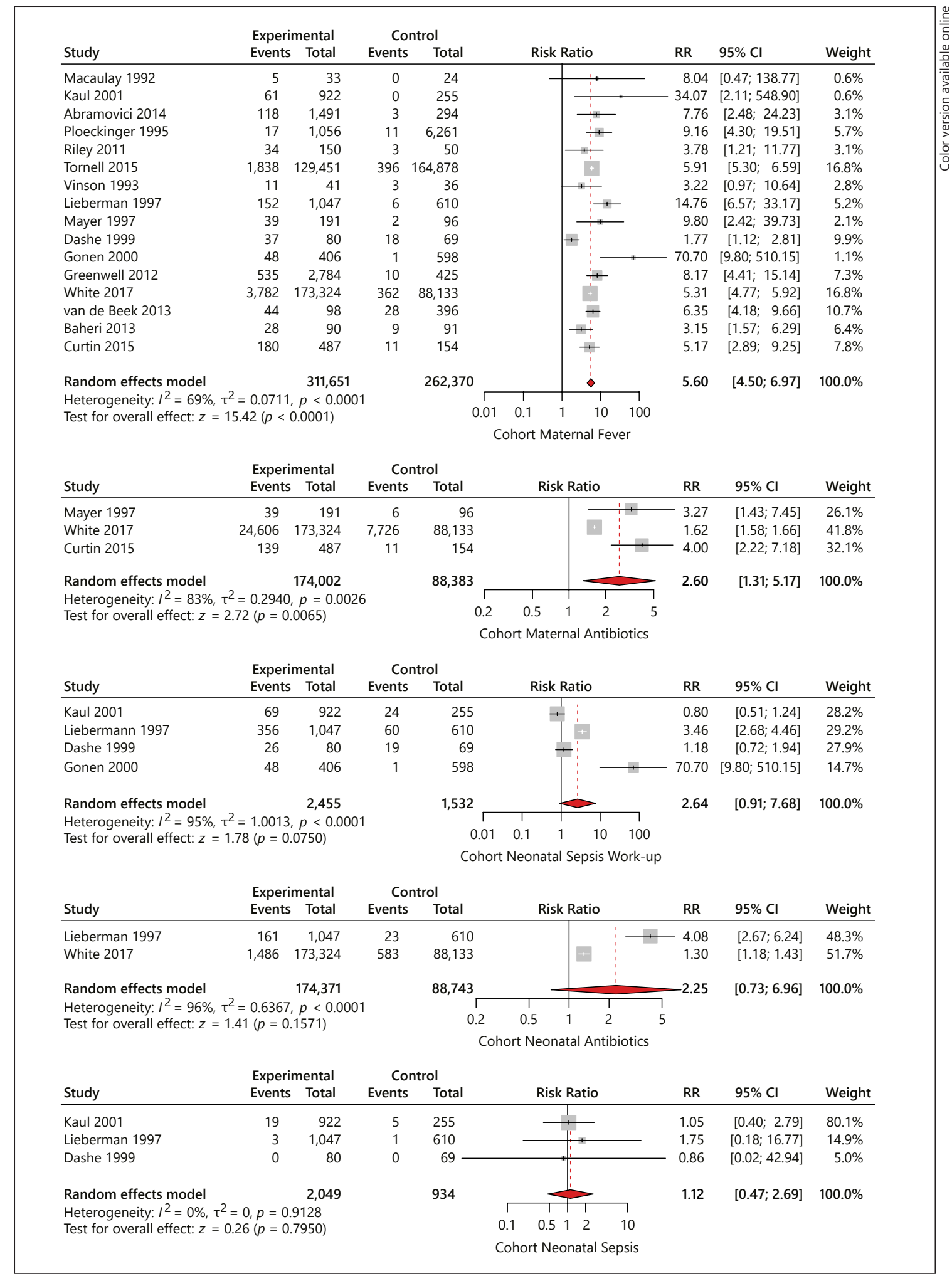

Fig. 3. Forest plots for observational cohort meta-analysis of primary and secondary outcomes epidural and nonepidural group. Size of gray boxes reflects precision or lack thereof of each individual study RR. Size of triangular random-effects summary measure reflects CI width. a Cohort maternal fever. b Cohort maternal antibiotics. c Cohort neonatal sepsis work-up. d Cohort neonatal antibiotics. e Cohort neonatal sepsis. RR, risk ratio. 
ing Egger's regression test revealed no evidence of publication bias (RCT maternal fever, $p=0.55$, cohort maternal fever, $p=0.65)$.

\section{Discussion}

\section{Main Findings}

We conducted a systematic review and meta-analysis to evaluate the effect of EA on adverse maternal and neonatal outcomes. Consistent with previous research [24, 25], we found an association between EA and intrapartum fever. Only within the meta-analysis of the observational cohort studies were mothers treated more often with antibiotics. The lack of an association between EA and neonatal bacteremia and antibiotic treatment can be explained by the limited power of our analysis due to the small number of inadequately conducted, biased studies with low numbers of occurrences. This precludes a reliable conclusion regarding the power of antibiotic treatment of the neonate for the prevention of a neonatal sepsis in the setting of epidural-related intrapartum fever.

\section{Interpretation}

While most discussions regarding epidural-related intrapartum fever focus on the potential presence of an intrapartum infection, whether intrapartum fever is of infectious origin remains unknown [25]. Various explanations postulating the role of other factors in the development of epidural-related intrapartum fever have emerged. Substantial evidence suggests that maternal inflammation is a factor. A study that investigated the association between EA and potential markers for intrapartum infection (i.e., maternal fever and placental inflammation) found that women in the epidural group were more likely to develop fever $\left(\geq 38^{\circ} \mathrm{C} ; 46 \mathrm{vs.} 26 \%\right)$ and have histological signs of placental inflammation [26]. However, since in the absence of placental inflammation there was no clear correlation between EA and intrapartum fever, fever in the absence of placental inflammation is not likely associated with EA. A study that investigated the role of noninfectious inflammatory factors in the development of epidural-related fever randomized afebrile nulliparous women with EA to receive either acetaminophen or a placebo [27]. Fever $\left(>38^{\circ} \mathrm{C}\right)$ occurred in $23.8 \%$ of all women and did not differ between the intervention and control groups. Nevertheless, increasing duration of EA was associated with increasing serum levels of maternal IL-6 and IL-8. Women who would develop fever were found to have higher IL- 6 levels at the time of admission compared with afebrile women and more likely to have additional risk factors for intrapartum infection (i.e., prolonged ruptured membranes, cervical examinations, etc.). These results suggest that women with an epidural and fever may already have an elevated inflammatory state prior to the epidural.

The method in which analgesia is administered is another potential factor in the development of maternal fever during labor. Mantha et al. [28] randomized women to receive either intermittent or continuous EA, including fentanyl for both groups. Women in the intermittent group had a lower incidence of fever $\left(\geq 38^{\circ} \mathrm{C}\right)$ in the first $4 \mathrm{~h}$ of labor compared with the continuous group (5 vs. $23 \%)$, although the effect was no longer significant thereafter. A possible explanation as given by the authors is that intermittent bolus administration of EA allowed for heat loss mechanisms to recover in between each injection and provided a protective effect against maternal fever during labor.

Inconsistency in obstetric management may influence the association between EA and intrapartum fever, even in adequately conducted clinical trials. In a secondary analysis of a RCT in which women were randomized to receive either EA or intravenous meperidine, women with fever $\left(\geq 38^{\circ} \mathrm{C}\right)$ were more likely to have prolonged labors, more internal fetal monitoring, and more oxytocin augmentation [29].

Duration of exposure to EA has also been suggested to be associated with the development of intrapartum fever. A study that investigated the effect of EA on the rate of maternal fever during labor, neonatal sepsis evaluations, and antibiotic treatment found that the proportion of women with fever increased from $7 \%$ for labors $<6 \mathrm{~h}$ in duration to $36 \%$ for labors $>18 \mathrm{~h}$ in duration after an epidural [30]. In contrast, the rate of fever in women without an epidural remained low independent of the length of labor [30]. Similarly, a more recent observational study [31], which investigated the effect of epidural-related maternal fever during labor on adverse maternal and neonatal outcomes found that women who had received an epidural for $\geq 6 \mathrm{~h}$ had a significantly higher risk of developing intrapartum fever compared to women who had received an epidural for $<6$ h (RR 5.23 vs. $1.73, p<0.001$ ) [31]. Moreover, exposure to EA for $\geq 6 \mathrm{~h}$ was associated with a higher risk of maternal antibiotic treatment, albeit there being no increased risk of adverse neonatal outcomes (i.e., Apgar score $<7$ at $1 \mathrm{~min}$, fetal distress, admission to neonatal ward, length of admission, assisted ventilation, neonatal infection, antibiotic treatment, and hyperbilirubinemia) [31]. Thus, these findings again support 
the potential role of noninfectious inflammation in the development of epidural-related maternal fever.

A final point of interest is the extent to which the magnitude of intrapartum fever is predictive of the risk of an underlying infectious etiology. Even though clinical and laboratory signs are more reliable predictors of serious illness than the height of fever, evidence has shown that hyperpyrexia is associated with more complicated disease [32]. In a study investigating the effect of different epidural-related temperature heights during labor on the risk of neonatal infection found that the neonatal infection risk increased from $4.9 \%$ in women with low-grade fever $\left(<38.5^{\circ} \mathrm{C}\right)$ to $10 \%$ in women with high-grade fever [33]. In the current meta-analysis, definitions of maternal fever during labor reported by the included studies varied between $\geq 37.5$ and $\geq 38^{\circ} \mathrm{C}$, without further specification on individual recorded temperatures. It is therefore plausible that the majority of parturients merely reached a maximum intrapartum temperature of $37.5-38^{\circ} \mathrm{C}$, with only a few women attaining temperatures of $\geq 40^{\circ} \mathrm{C}$ and thereby having a higher risk of a potential underlying infection. Identifying a threshold for epidural-related intrapartum hyperthermia above which a potential risk of maternal and/or neonatal infection is increased could help limit unnecessary exposure to antibiotic treatment.

\section{Strengths and Limitations}

To our best knowledge, this is the first meta-analysis that has comprehensively evaluated the effect of epiduralrelated intrapartum fever on both maternal and neonatal outcomes simultaneously. To increase the reliability of our results, we also conducted separate meta-analyses for each study type. Nevertheless, some limitations in our systematic review still remain. First, most evidence for the correlation between EA and maternal fever during labor is derived from retrospective observational studies in which EA was given upon request. Many of these studies are thus limited by selection bias [34], as women who choose EA may already be inherently at risk for the development of intrapartum fever due to factors including nulliparity, prolonged duration and/or difficulty of labor, premature rupture of the membranes, and increased intrapartum cervical examinations $[24,35,36]$. The most appropriate way to isolate the effect of EA would be by performing an RCT or by correcting for potential confounding factors using multivariate logistic regression. Second, we were unable to evaluate the association between EA and maternal infection due to the lack of a consensus definition for maternal infection among the included studies. Although maternal infection is generally documented by positive cultures of biologic fluids (blood and amniotic fluid), most studies either equated maternal fever to chorioamnionitis or relied upon the presence of placental inflammation without the performance of bacterial cultures. Moreover, some evidence has even suggested the possible presence of "sterile chorioamnionitis" or placental inflammation without infection. A secondary analysis of low-risk women found that the association between fever and histologic chorioamnionitis was present in women with and without EA [37]. The overall rate of infection among those with histologic chorioamnionitis was only $5 \%$, supporting the role for a noninfectious inflammatory process. The diverging definitions of maternal infection between studies may explain the frequent initiation of antibiotic treatment based on suspicion rather than objectified markers of infection. Similarly, the significant incongruity among studies regarding the definition of neonatal sepsis may explain the large variation in treatment management of the neonate. Even though the "gold standard" for diagnosing neonatal sepsis is the presence of a positive blood culture, only 3 studies [16, 30, 38] implemented this diagnostic measure, while the remaining studies $[18,21,26]$ either relied upon nonspecific clinical signs and symptoms (i.e., "suspected" sepsis) or did not mention the diagnostic procedure. Third, a substantial amount of protocol noncompliance was reported for the nonepidural groups in several RCTs $[13-18,20]$. Because cross-overs commonly occur as a result of inadequate pain relief due to complicated labors, this may have led to an overestimation of the association between EA and intrapartum fever. Finally, the majority of the included studies are underpowered, yielding statistically weak results and considerable heterogeneity for most secondary outcomes. In particular, although no infant developed a bacteremia in the RCT analysis, 19 of the 22 infants who developed a bacteremia in the cohort analysis derived from only 1 study [38], illustrating the lack of power of this analysis to draw a definite conclusion.

Until now, epidural-related maternal fever remains a poorly understood physiologic phenomenon, even though its postpartum clinical management necessitates proper attention. While some studies indicate that intrapartum fever is associated with clinical depression of the neonate, including hypotonia, low Apgar scores, and the need for assisted ventilation, there has been no documented increased risk for neonatal sepsis [39]. Given our limited ability to reliably distinguish the need to initiate and cease antibiotic treatment, it is plausible that overexposure to antibiotics is rising, with concomitant increases in antibiotic resistance and health care costs. Although we 
were not able to conclude that there was no neonatal bacteremia as a result of EA, prudent antibiotic treatment and more accurate prediction models of neonates at risk for sepsis are warranted.

\section{Conclusion}

This systematic review demonstrates that EA is associated with intrapartum fever. Although an increased risk of maternal and neonatal bacteremia was not observed, the quality of evidence was insufficient for all investigated outcomes. Nevertheless, without confirmation of a true infectious inflammatory process, neonatal sepsis evaluations and antibiotic treatment may be avoidable. A large RCT investigating the effectiveness of antibiotic treatment in the prevention of maternal and neonatal bacteremia in the setting of epidural-related intrapartum fever is therefore warranted. Adjustments to current obstetric and pediatric protocols can be made by identifying the appropriate diagnostic criteria in a clinically relevant time frame for the diagnosis and treatment of neonatal sepsis.

\section{Acknowledgments}

None.

\section{Statement of Ethics}

The authors have no ethical conflicts to disclose.

\section{Disclosure Statement}

The authors have no conflicts of interest to declare.

\section{Author Contributions}

S.J. drafted the protocol and manuscript; acquired, analyzed, and interpreted the data; and provided final approval for publication. E.L. helped analyze and interpret the data, revised the manuscript for important intellectual content, provided final approval for publication, and agreed to be accountable for all aspects of the work. C.N. contributed to the conception and design of the work, revised the manuscript for important intellectual content, helped analyze and interpret the data, and provided final approval for publication. J.L. constructed the search, contributed to the conception and design of the work, revised the manuscript for important intellectual content, and provided final approval for publication. M.S. helped analyze and interpret the data, revised the manuscript for important intellectual content, and provided final approval for publication. E.L. initiated the project, contributed to the conception and design of the work, revised the manuscript for important intellectual content, and provided final approval for publication. V.B. initiated the project; contributed to the conception and design of the work; drafted the protocol; acquired, analyzed, and interpreted the data; revised the manuscript for important intellectual content; and provided final approval for publication.

\section{References}

1 Apantaku O, Mulik V. Maternal intra-partum fever. J Obstet Gynaecol. 2007 Jan;27(1):12-5.

2 Alexander JM. Epidural analgesia for labor pain and its relationship to fever. Clin Perinatol. 2005 Sep;32(3):777-87.

3 National Institute for Health and Excellence (NICE). Neonatal infection (early onset): antibiotics for prevention and treatment: NICE Clinical Guideline [CG149] [Internet]. Manchester, United Kingdom: National Institute for Health and Excellence; 2012 [updated 2012 October]. Available from: https://www. nice.org.uk/guidance/CG149 [Accessed 31st December 2018].

4 Gerdes JS, Polin R. Early diagnosis and treatment of neonatal sepsis. Indian J Pediatr. 1998 Jan-Feb;65(1):63-78.

5 Liberati A, Altman DG, Tetzlaff J, Mulrow C, Gøtzsche PC, Ioannidis JP, et al. The PRISMA statement for reporting systematic reviews and meta-analyses of studies that evaluate healthcare interventions: explanation and elaboration. BMJ. 2009 Jul;339 jul21 1:b2700.
6 Cochrane Consumers and Communication Review Group. Heterogeneity and subgroup analyses in Cochrane consumers and communication group reviews: planning the analysis at protocol stage [Internet]. 2016. Available from: http://cccrg.cochrane.org/sites/ cccrg.cochrane.org/files/public/uploads/heterogeneity_subgroup_analyses_revising december_1st_2016.pdf.

7 Cochrane Database of Systematic Reviews. A revised tool for assessing risk of bias in randomized trials [Internet]. [Accessed December 31, 2018]. Available from: https://sites. google.com/site/riskofbiastool/welcome/ rob-2-0-tool.

8 National Heart, Lung, and Blood Institute. Study Quality Assessment Tools. Quality Assessment Tool for Observational Cohort and Cross-Sectional Studies. [Accessed December 31, 2018]. Available from: https://www.nhlbi. nih.gov/health-topics/study-quality-assessment-tools.
9 The GRADE Working Group. GRADE handbook for grading quality of evidence and strength of recommendations [Internet]. 2013 [updated 2013 October]. [Accessed 31st December 2018]. Available from: https://gdt. gradepro.org/app/handbook/handbook. html.

10 Guyatt G, Oxman AD, Akl EA, Kunz R, Vist G, Brozek J, et al. GRADE guidelines: 1. Introduction-GRADE evidence profiles and summary of findings tables. J Clin Epidemiol. 2011 Apr;64(4):383-94.

11 Riley LE, Celi AC, Onderdonk AB, Roberts DJ, Johnson LC, Tsen LC, et al. Association of epidural-related fever and noninfectious inflammation in term labor. Obstet Gynecol. 2011 Mar;117(3):588-95.

12 Abramovici A, Szychowski JM, Biggio JR, Sakawi Y, Andrews WW, Tita AT. Epidural use and clinical chorioamnionitis among women who delivered vaginally. Am J Perinatol. 2014 Nov;31(11):1009-14.
Epidural-Related Intrapartum Fever and Maternal and Neonatal Morbidity 
13 Logtenberg S, Oude Rengerink K, Verhoeven CJ, Freeman LM, van den Akker E, Godfried $\mathrm{MB}$, et al. Labour pain with remifentanil patient-controlled analgesia versus epidural analgesia: a randomised equivalence trial. BJOG. 2017 Mar; 124(4):652-60.

14 Freeman LM, Bloemenkamp KW, Franssen MT, Papatsonis DN, Hajenius PJ, Hollmann MW, et al. Patient controlled analgesia with remifentanil versus epidural analgesia in labour: randomised multicentre equivalence trial. BMJ. 2015 Feb;350 feb23 10:h846.

15 Lucas MJ, Sharma SK, McIntire DD, Wiley J, Sidawi JE, Ramin SM, et al. A randomized trial of labor analgesia in women with pregnancy-induced hypertension. Am J Obstet Gynecol. 2001 Oct;185(4):970-5.

16 Douma MR, Stienstra R, Middeldorp JM, Arbous MS, Dahan A. Differences in maternal temperature during labour with remifentanil patient-controlled analgesia or epidural analgesia: a randomised controlled trial. Int $\mathrm{J} \mathrm{Ob}$ stet Anesth. 2015 Nov;24(4):313-22.

17 Sharma SK, Alexander JM, Messick G, Bloom SL, McIntire DD, Wiley J, et al. Cesarean delivery: a randomized trial of epidural analgesia versus intravenous meperidine analgesia during labor in nulliparous women. Anesthesiology. 2002 Mar;96(3):546-51.

18 Ramin SM, Gambling DR, Lucas MJ, Sharma SK, Sidawi JE, Leveno KJ. Randomized trial of epidural versus intravenous analgesia during labor. Obstet Gynecol. 1995 Nov;86(5):7839.

19 Gambling DR, Sharma SK, Ramin SM, Lucas MJ, Leveno KJ, Wiley J, et al. A randomized study of combined spinal-epidural analgesia versus intravenous meperidine during labor: impact on cesarean delivery rate. Anesthesiology. 1998 Dec;89(6):1336-44.

20 Sharma SK, Sidawi JE, Ramin SM, Lucas MJ, Leveno KJ, Cunningham FG. Cesarean delivery: a randomized trial of epidural versus patient-controlled meperidine analgesia during labor. Anesthesiology. 1997 Sep;87(3):48794.

21 de Orange FA, Passini R Jr, Amorim MM, Almeida T, Barros A. Combined spinal and epidural anaesthesia and maternal intrapartum temperature during vaginal delivery: a randomized clinical trial. Br J Anaesth. 2011 Nov; 107(5):762-8.

22 Törnell S, Ekéus C, Hultin M, Håkansson S, Thunberg J, Högberg U. Low Apgar score, neonatal encephalopathy and epidural analgesia during labour: a Swedish registry-based study. Acta Anaesthesiol Scand. 2015 Apr; 59(4):486-95.
23 Mayer DC, Chescheir NC, Spielman FJ. In creased intrapartum antibiotic administration associated with epidural analgesia in labor. Am J Perinatol. 1997 Feb;14(2):83-6.

24 Heesen M, Klöhr S, Rossaint R, Straube S, Van de Velde M. Labour epidural analgesia and anti-infectious management of the neonate: a meta-analysis. J Perinat Med. 2012 Nov;40(6): 625-30.

25 Leighton BL, Halpern SH. The effects of epidural analgesia on labor, maternal, and neonatal outcomes: a systematic review. Am J Obstet Gynecol. 2002 May;186(5 Suppl Nature):S69-77.

26 Dashe JS, Rogers BB, McIntire DD, Leveno $\mathrm{KJ}$. Epidural analgesia and intrapartum fever: placental findings. Obstet Gynecol. 1999 Mar; 93(3):341-4.

27 Goetzl L, Evans T, Rivers J, Suresh MS, Lieberman E. Elevated maternal and fetal serum interleukin-6 levels are associated with epidural fever. Am J Obstet Gynecol. 2002 Oct;187(4):834-8.

28 Mantha VR, Vallejo MC, Ramesh V, Phelps AL, Ramanathan $S$. The incidence of maternal fever during labor is less with intermittent than with continuous epidural analgesia: a randomized controlled trial. Int J Obstet Anesth. 2008 Apr;17(2):123-9.

29 Philip J, Alexander JM, Sharma SK, Leveno KJ, McIntire DD, Wiley J. Epidural analgesia during labor and maternal fever. Anesthesiology. 1999 May;90(5):1271-5.

30 Lieberman E, Lang JM, Frigoletto F Jr, Richardson DK, Ringer SA, Cohen A. Epidural analgesia, intrapartum fever, and neonatal sepsis evaluation. Pediatrics. 1997 Mar;99(3): 415-9.

31 Yin $\mathrm{H}, \mathrm{Hu}$ R. A cohort study of the impact of epidural analgesia on maternal and neonatal outcomes. J Obstet Gynaecol Res. 2019 Aug; 45(8):1435-41.

32 Barbi E, Marzuillo P, Neri E, Naviglio S, Krauss BS. Fever in children: pears and pitfalls. Children (Basel). 2017 Sep;4(9):81.

33 Henriksen TB, Hermansen S, Wisborg K, Hansen AK. Newborn infection: should low grade fever associated with epidural analgesia increase the awareness at all? ADC. 2008;93: 229.

34 Lieberman E. No free lunch on labor day. The risks and benefits of epidural analgesia during labor. J Nurse Midwifery. 1999 Jul-Aug;44(4): 394-8.

35 Macaulay JH, Bond K, Steer PJ. Epidural analgesia in labor and fetal hyperthermia. Obstet Gynecol. 1992 Oct;80(4):665-9.
36 Vinson DC, Thomas R, Kiser T. Association between epidural analgesia during labor and fever. J Fam Pract. 1993 Jun;36(6):617-22.

37 Roberts DJ, Celi AC, Riley LE, Onderdonk AB, Boyd TK, Johnson LC, et al. Acute histologic chorioamnionitis at term: nearly always noninfectious. PLoS One. 2012;7(3):e31819.

38 Kaul B, Vallejo M, Ramanathan S, Mandell G. Epidural labor analgesia and neonatal sepsis evaluation rate: a quality improvement study. Anesth Analg. 2001 Oct;93(4):986-90.

39 Greenwell EA, Wyshak G, Ringer SA, Johnson LC, Rivkin MJ, Lieberman E. Intrapartum temperature elevation, epidural use, and adverse outcome in term infants. Pediatrics. 2012 Feb;129(2):e447-54.

40 Evron S, Ezri T, Protianov M, Muzikant G, Sadan O, Herman A, et al. The effects of remifentanil or acetaminophen with epidural ropivacaine on body temperature during labor. $J$ Anesth. 2008;22(2):105-11.

41 Nafisi S. Effects of epidural lidocaine analgesia on labor and delivery: a randomized, prospective, controlled trial. BMC Anesthesiol. 2006 Dec;6(1):15.

42 Evron S, Parameswaran R, Zipori D, Ezri T, Sadan O, Koren R. Activin betaA in term placenta and its correlation with placental inflammation in parturients having epidural or systemic meperidine analgesia: a randomized study. J Clin Anesth. 2007 May;19(3):168-74.

43 Ploeckinger B, Ulm MR, Chalubinski K, Gruber W. Epidural anaesthesia in labour: influence on surgical delivery rates, intrapartum fever and blood loss. Gynecol Obstet Invest. 1995;39(1):24-7.

44 Gonen R, Korobochka R, Degani S, Gaitini L. Association between epidural analgesia and intrapartum fever. Am J Perinatol. 2000; 17(3):127-30.

45 White A, Olson D, Messacar K. A state-wide assessment of the association between epidural analgesia, maternal fever and neonatal antibiotics in Colorado, 2007-2012. Arch Dis Child Fetal Neonatal Ed. 2017 Mar; 102(2):F120-5

46 van de Beek RC, Sporken JM, van Heijst AF, Semmekrot BA. Epidural analgesia and maternal during labor. Tijdschr Kindergeneeskd. 2013;81(4):82-8.

47 Baheri B, Coppejans H, Joukes E, Vercauteren $M$. Do epidurals cause higher intrapartum temperatures in parturients and neonates? A Belgian experience. J Rom Anest Terap Int. 2013;20(1):10-6.

48 Curtin WM, Katzman PJ, Florescue H, Metlay LA, Ural SH. Intrapartum fever, epidural analgesia and histologic chorioamnionitis. J Perinatol. 2015 Jun;35(6):396-400. 